\title{
Impact of the COVID-19 pandemic on orthopedic surgeon in Pakistan
}

\author{
Muhammad Muzzammil ${ }^{1 *}$, Syed Jahanzeb², Ali Asghar³ ${ }^{3}$, Saadia Jabbar ${ }^{4}$, Hira Waheed ${ }^{5}$
}

\author{
${ }^{1}$ Department of Orthopedics, Sindh Gov. Services Hospital, Karachi, Pakistan \\ ${ }^{2}$ Department of Orthopedics, Dr Ruth K. M. Pfau Civil Hospital, Karachi, Pakistan \\ ${ }^{3}$ Department of Surgery, Lyari General Hospital, Karachi \\ ${ }^{4}$ Ziauddin Medical University, Karachi, Pakistan \\ ${ }^{5}$ Aga Khan University, Karachi, Pakistan
}

Received: 07 August 2020

Revised: 10 October 2020

Accepted: 12 October 2020

\section{*Correspondence:}

Dr. Muhammad Muzzammil,

E-mail: muzzammil_sangani@hotmail.com

Copyright: ( ) the author(s), publisher and licensee Medip Academy. This is an open-access article distributed under the terms of the Creative Commons Attribution Non-Commercial License, which permits unrestricted non-commercial use, distribution, and reproduction in any medium, provided the original work is properly cited.

\section{ABSTRACT}

Background: The objective of our study is to assess the impact of COVID-19 pandemic on clinical practice as well as psychological impact on orthopedic surgeons of Pakistan.

Method: An online survey involving orthopedic surgeons through social media platforms like WhatsApp and Facebook and also through email, to assess the impact of COVID-19 pandemic. Our survey also aims to evaluate their state of mind and physical as well as emotional wellbeing in the wake of COVID-19 pandemic.

Results: Among 77 respondents, mostly 31 (40.3\%) were consultant and mostly $28(36.4 \%)$ having experience in field of orthopedics was between 5-10 years. This data revealed $48(62.3 \%)$ respondents did not received any specific training or recommendations about COVID pandemic from their institute and only $37(48.1 \%)$ reported they are well informed on latest COVID guidelines. Interestingly, this data revealed the willingness of orthopedic surgeons 30 (39\%) to take care of COVID-19 positive patients in ICU.

$67(87 \%)$ feel anxious, stressed, furious, angry or depressed due to pandemic and most common fear among them was to infect their family members followed by getting infected and loss of their life and health. $40(51.9 \%)$ did not think their institute is well equipped to deal COVID pandemic.

Conclusions: Orthopedic community nevertheless have to play an important role in the face of an increasing psychological and emotional stress which comes from working in a post COVID era. The dread of getting infected while treating and the sinking feeling of spreading the contagion to their loved ones has put a massive toll on the emotional well-being and needs to be addressed through open and fair discussion

Keywords: COVID-19, Orthopedic surgeon, Pandemic, Stress, Psychological impact

\section{INTRODUCTION}

COVID-19 pandemic is gearing up to be the most devastating global healthcare crisis ever observed in nearly 100 years. It is a disease caused by the novel corona virus, which was initially called 2019-nCoV, consequently termed as SARS-CoV-2 by the experts of the international committee on taxonomy of viruses (ICTV). ${ }^{1}$
This novel corona virus was first identified in Wuhan, the largest urban area in China's Hubei province, and was first reported to the WHO country office in China, on December 31, 2019. ${ }^{1}$ WHO already had declared COVID19 as an international emergency as the disease is spreading at alarming levels affecting 199 countries and territories across the world. ${ }^{2}$ 
To combat this rapidly spreading catastrophic virus, which has already collapsed major healthcare systems around the world including Europe and China, health departments around the world are rushing in to devise various protocols to combat the pandemic and at the same time protect their healthcare staff from the contagion.

Orthopedic surgeons are not usually considered as frontline fighters in the on-going struggle against COVID-19 as compared to the healthcare providers working in the departments that are facing this pandemic in a closer proximity for e.g. intensivists, pulmonologists and ICU staff etc. But there is no denying the fact that orthopedic surgeons are in fact part of the ongoing struggle against COVID-19 because they continually have to manage trauma related patients and cannot just sit at home and quarantine themselves from the pandemic. ${ }^{3}$

In the light working under the constant threat of getting infected while treating orthopedic related patients, and trying to minimize their exposure as much as possible to avoid the contagion, has stressed out even the more seasoned orthopedic.

So, we decided to initiate an anonymous online survey, and our target was to solely involve the orthopedic surgeons of our country to figure out how much this ongoing pandemic has affected their mental well-being, how prepared they feel they are to face this threat and what, according to them in the end-game solution to this pandemic.

\section{METHODS}

A questionnaire was made and anonymity of participants was made sure. It was communicated to them through email and other social media platforms. An online survey generator was used to make the questionnaire and only orthopedic surgeons of Pakistan were involved in this survey. The questionnaire was designed to assess the effects of COVID-19 pandemic on orthopedic surgeons' day to day practice and its impact on their mental wellbeing. How well equipped they feel they are both in terms of knowledge and availability of PPE's, to combat this pandemic was also taken into account. The respondents were required to report their experience of care for COVID-19 positive patients and their motivation to work in COVID-19 units. Questions were asked specifically about their orthopedics and trauma endeavors: number of surgeries (planned or emergency), number of outpatient visits and supply of orthopedic equipment. They were also inquired about any possibly signs and symptoms they might have experienced and what would their line of action be if they did. Questions were also asked about their willingness to work in a COVID-19 unit if the need arises. Also, if they are willing to adopt modern methods of consultation like telemedicine and video conferencing. Availability of PPE's was also inquired and if they can readily get their hands-on PPE's easily at their institution. And how do they think COVID-19 spread should be dealt with. The respondents were also asked about their institute strategy to combat COVID-19 pandemic and if they agree with it or not.

A questionnaire based anonymous survey was sent by email and social media platform to orthopedic surgeons practicing in different parts of Pakistan. We developed an online, web-based survey using an online survey generator. The questionnaire was designed to assess the effect of the COVID-19 pandemic on the orthopedic surgeons' activities and its psychological impact. We distributed the questionnaire by e-mail and collected relevant data. The survey was conducted within 5 days to eliminate repetition and bias due to information spreading about the questionnaire content. Confidentiality of the respondents was ensured and maintained by the researchers. Results were exported to Microsoft excel version 2010 for analysis.

The survey included different sections. The first one was about general information: area of practice, age, sector of activity and years of experience. Then, we asked about knowledge about the COVID-19: specific training, recommendations, risks and protection. The respondents were required to report their experience of care for COVID-19 positive patients and their motivation to work in COVID-19 units. Specific questions were asked about orthopedic and trauma activity: number of surgeries (planned or emergency), number of outpatient visits and supply of orthopedic equipment. They were also interrogated if they had reported symptoms or had suspected a COVID-19 infection and what were the measures taken about that. They were also required to report the level of personal protection at work, which equipment they used and which protection equipment is missing the most in their institution. The respondents were also asked about their institute strategy and what do they think is the best solution to face the COVID-19 pandemic. Finally, questions were asked about psychological stress on them.

\section{RESULTS}

Among 77 respondents, mean age of participants was $47 \pm 12$ years, $31(40.3 \%)$ were consultant, $18(23.4 \%)$ were assistant professors and 11 were $(14.3 \%)$, mostly working in public sector hospitals $51(66.2 \%)$. Mostly $28(36.4 \%)$ having experience in field of orthopedics was between 510 years followed by $25(32.5 \%)$ having experience between 1-5 years.

This data revealed $48(62.3 \%)$ respondents did not received any specific training or recommendations about COVID pandemic from their institute and principle source of knowledge among them was social media $46(59.7 \%)$ followed by WHO press release $14(18.2 \%)$ and $10(13 \%)$ from their institutes. Only $37(48.1 \%)$ reported they are well informed on latest COVID-19 guidelines while 28 $(36.4 \%)$ were somewhat informed. $61(79.2 \%)$ reported they are aware of measures to protect themselves and patient during pandemic and $11(14.3 \%)$ were not sure. 
During pandemic 73 (94.8\%) did not operate any COVID19 patient and among them $31(40.3 \%)$ not declared willingness to do elective orthopedic surgery on COVID19 positive patient. Interestingly, this data revealed the willingness of orthopedic surgeons 30 (39\%) to take care of COVID-19 positive patients and support their colleagues in intensive care units, if needed.

Concerning their orthopedic practice, 35 (45.5\%) stopped planned surgeries while $17(22.1 \%)$ decrease their practice by $90 \% .20(26 \%)$ reported decrease in emergency surgeries during pandemic while 19 (24.7\%) did not report any change. This might be due to decrease in road traffic accident notified by study participant 24 (31.2\%). 26 $(33.8 \%)$ reported reduced orthopedic consultation by $50 \%$ while $18(23.4 \%)$ reduced by $75 \%$. $46(59.7 \%)$ reported decrease in number and quantity of orthopedic equipment supply during pandemic. For consultation 56 (72.7\%) respondent used telemedicine and video conference during pandemic.

During pandemic respondent revealed that in suspicious of COVID-19 infection mostly 34 (44.2\%) agreed on go through COVID-19 test and isolation while 28 (36.4\%) agreed to stay at home and observe and $65(84.4 \%)$ agreed on complete isolation on positive testing.

67 (87\%) feel anxious, stressed, furious, angry or depressed due to pandemic and most common fear among them was to infect their family members followed by getting infected and loss of their life and health. As age decreased, percentage of orthopedic surgeons feeling stressed out increased. Only $4(5.8 \%)$ estimate their protection, protected while $41(53.2 \%)$ reported average protection. On personal safety $71(92.2 \%)$ use medical mask while $39(50.6 \%)$ have facility of N95 mask, 20 (26\%) use face shields, 66 (85.7\%) use gloves, 43 (55.8\%) gowns and $29(37.7 \%)$ have protective glass facility.

To avoid COVID 19 mostly agreed to advise their patients for regular hand wash $76(98.7 \%)$, sanitizers $73(94.8 \%)$, social distancing $73(94.8 \%)$, facemask $74(96.1 \%)$ and stay home $72(93.5 \%)$.

\section{Province Name}

77 responses

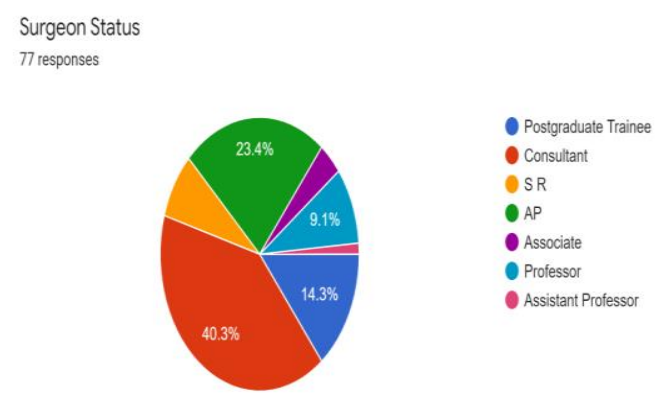

Figure 2: Demography of study participant by surgeon status.

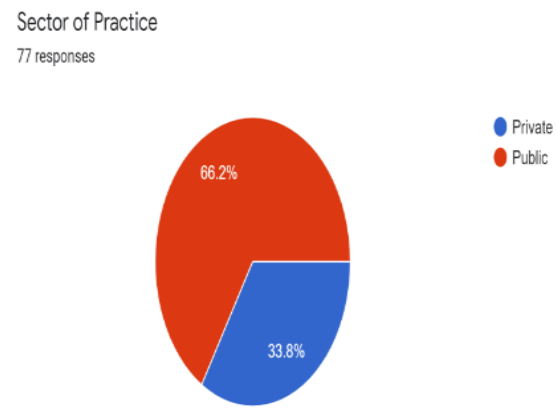

Figure 3: Demography of study participant by sector of practice.

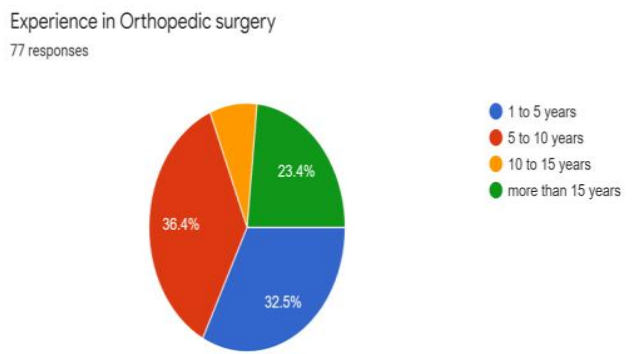

Figure 4: Demography of study participant by experience in orthopedic surgery.

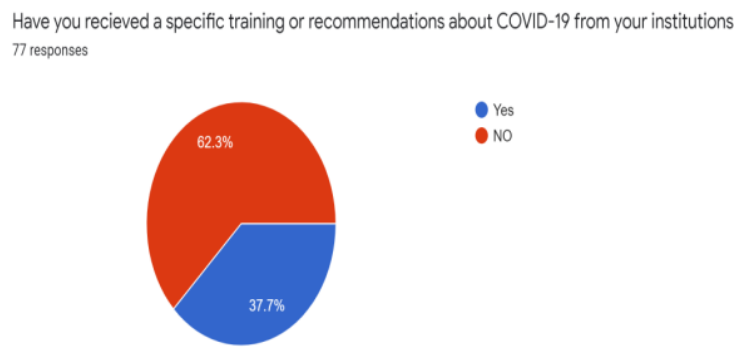

Figure 1: Demography of study participant by province name.
Figure 5: Responses of study participants about training or recommendations about COVID-19.

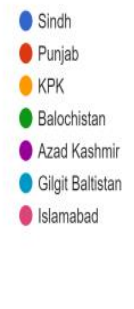

International Journal of Research in Orthopaedics | January-February 2021 | Vol 7 | Issue 1 Page 14 
What is the principal source of knowledge about COVID-19

77 responses

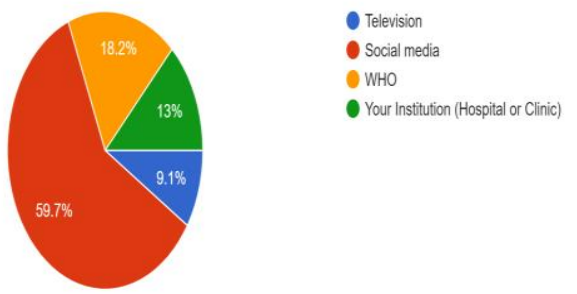

Figure 6: Responses of study participants about source of knowledge about COVID-19.

Do you feel well informed on the latest COVID-19 guidelines 77 responses

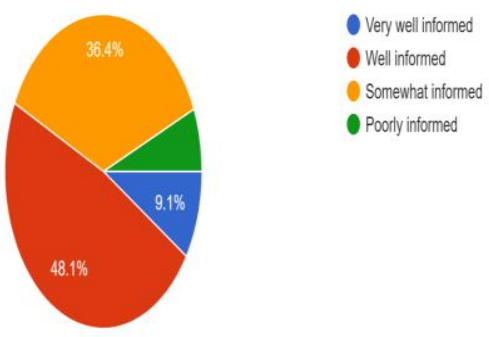

Figure 7: Responses of study participants about information guidelines.

Are you willing to operate COVID-19 positive patients with an orthopedic pathology 77 responses

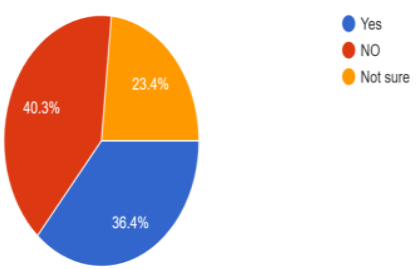

Figure 8: Responses of study participants about operating COVID-19 positive patients.

Are you willing to work and help in intensive care units if necessary 77 responses
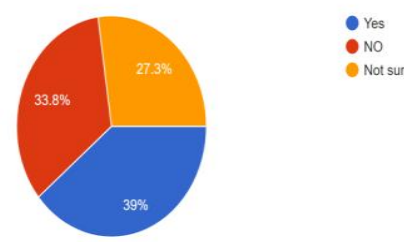

Figure 9: Responses of study participants about working in ICU if necessary.
With the COVID-19 pandemic, the number of planned surgeries you are practicing 77 responses
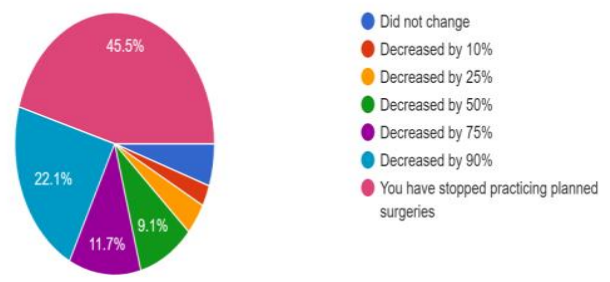

Figure 10: Responses of study participants about the number of planned surgeries practicing.

During COVID-19 pandemic, the number of emergency surgeries (trauma) you are practicing 77 responses
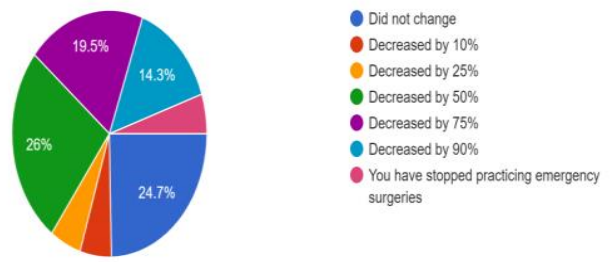

Figure 11: Responses of study participants about the number of emergency surgeries (trauma) you are practicing during COVID-19 pandemic.

With the COVID-19 pandemic, have you used telemedicine, video conference or phone consultation with your patients 77 responses

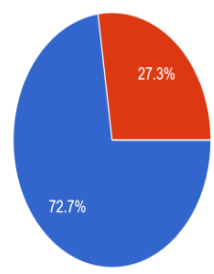

Yes

Figure 12: Responses of study participants about use of telemedicine, video conference or phone consultation with patients.

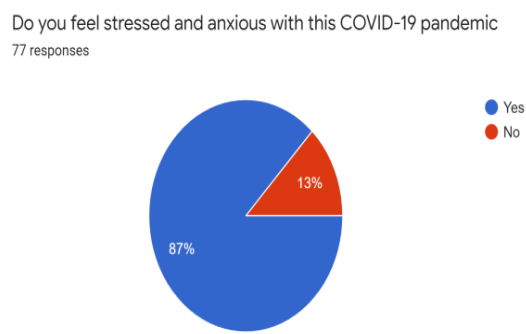

Figure 13: Responses of study participants about stress and anxiety with COVID-19 pandemic. 
Do you think that your institute is well equipped to deal with this COVID-19 pandemic 77 responses

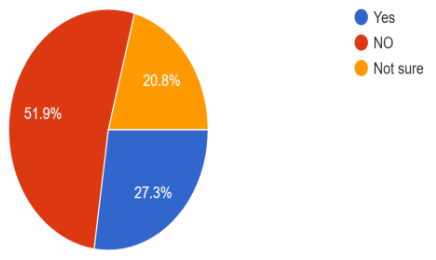

Figure 14: Responses of study participants about institutional equipment to deal with COVID-19 pandemic.

$40(51.9 \%)$ did not think their institute is well equipped to deal COVID-19 pandemic and 31 (40.3\%) did not agreed to strategy of their institute to face COVID-19 pandemic. Mostly agreed on specific vaccine 25 (32.5\%) quarantine $26(33.8 \%)$ would be best solution to preventive method to handle pandemic.

\section{DISCUSSION}

SARS-CoV-2 pandemic is proving to be a very formidable challenge for healthcare professionals. ${ }^{4}$ Its rampant and unrelenting spread poses a massive impact on practicing healthcare workers. We took onboard various orthopedic surgeons from different regions of Pakistan to take part in our study.

The aim of this study was to assess the impact of COVID19 on the day to day practice of orthopedic surgeons across the country. The mean age of participants was $47 \pm 12$ years. The source of information used by healthcare professionals is not only limited to what is shared by their respective institutions but also from social media, print material, WHO website. This study provided us with the data that showed a high level of awareness among practicing orthopedic surgeons about the risk of infection for them as well as the patients and how to limit the spread of this disease using a lot of safety measures at their disposal.

Risk of COVID-19 transmission increases multiple folds in hospitals and especially in operation theatres, so specialized personal protective equipment must be provided. As Surgeons, it is our responsibility to take every step necessary to make sure no contamination or spread of the disease happens preoperatively and also in postoperative settings. Risk and benefits should always be rationalized in each patient as the COVID-19 has shown increased infectivity and a higher fatality rate than H1N1 virus. $^{2,3,5-7}$ Additionally vital clinical features of COVID19 are currently unknown which points towards the fact that $(40.3 \%)$ of the interviewees expressed reluctance to treat or surgically manage non emergent conditions in COVID-19 positive patients, because of an unnecessary risk of getting infected themselves or transmitting the infection to their family members. So, with a better understanding of the nature and characteristics of COVID19 , we should expect a gradual increase in the number of medical professionals who will be willing to treat and manage the infected patients. ${ }^{2.5}$ Since the dawn of COVID19 pandemic we have seen health professionals working outside of their specialty, providing support to infections, pulmonologists and intensivists. ${ }^{1,8}$ Orthopedic practice has also taken a blow after the advent of this COVID outbreak, largely guided by urgency of clinical care provision, protection of patients and the medical staff and conservation of health-care resources. With these points in mind, changes to inpatient and outpatient care have been set in motion to curb the spread of the contaminant among patients and medical staff, and to also enable the hospital to free up precious resources as in beds in wards and ICU's for the treatment of COVID-19 positive or suspected patients. ${ }^{3,8}$

After to emergence of such a pandemic, we need to reflect and re-evaluate the use of contemporary means of managing our patients which includes telemedicine and tele-rehabilitation initiatives, which will allow us to consult and follow-up with the patients in the comfort of their homes. ${ }^{3,8,10-12}$ Although it is known the COVID-19 virus likely causes minor symptoms in majority (more than $80 \%$ ) of the infected people and a lot of healthcare workers are likely to fall in that category, during such difficult times emotional and psychological support is very important and should be always provided where needed, to healthcare workers as well as their family, friends, colleagues and patients. Making available all the necessary personal protective equipment to healthcare professionals has been a herculean task for many countries. ${ }^{1,9,13}$ PPE's include medical masks, respirator N-95 or FFP2 masks, face shields, gloves, gowns, and protective glasses. ${ }^{5,14,15}$ With the rampant pandemic going on, a shortage of PPE's specially FFP2 mask sits on top of the list-reported by $50.6 \%$ of the interviewed participants. Government bodies and various industries have set in motion different strategies to counter this shortage of PPE's by increasing the manufacturing and approval of development and provision to healthcare institutions during this pandemic. $^{15,16}$ To combat the spread of COVID-19 orthopedic surgeons recommend frequent hand washing for at least 20 seconds, use hydro-alcoholic sanitizers, adequate social distancing, use of face masks, gloves and every possible safety measure until a specific treatment or a vaccine is not produced for this pandemic.

Importantly, a number of orthopedic surgeons who felt they were stressed out had a feeling that their professional life will never be the same again or it would be a challenge to bring it back to normal. This stress comes due of a number of factors such as family health risk, loss of surgical practice and acumen, financial burden or the anxiety that eventually they may be asked to work as a frontline care-giver. ${ }^{17}$

Other concerns were of risking the life and health of their family members and unknowingly getting infected with 
contagion from workplace as a study by Guo et al showed that 26 orthopedic surgeons were infected by COVID-19 during patient care activity. ${ }^{18}$ Rate at which family members of healthcare providers got infected with it was high and this fact caused massive psychological stress to the orthopedic surgeons. Anger, irritability, high anxiety levels and acute stress disorder has been associated with quarantine of healthcare workers. ${ }^{19}$

It is in time like these that we as a community of healthcare providers must rise to the occasion and fight this pandemic with close cooperation, teamwork, collaborative efforts and open communication and voicing of concerns, specially from younger members of fraternity. Hopefully soon we will emerge from these dark times of COVID-19 pandemic with much more resilience and vigor.

\section{Limitation}

Our study does not come without its limitations. For instance, the extent and severity of COVID-19 outbreak has not been the same in all countries so the measures taken by the government and health institutions may vary from country to country. ${ }^{6,7}$ Secondly, the experience of the person filling out the questionnaire regarding COVID-19 may affect the results. however, survey was totally anonymous and made sure to hide identity of orthopedic who filled it. This leads us to believe that participants were truly honest in declaring how they were feeling.

\section{CONCLUSION}

COVID-19 pandemic has pitted our orthopedic community against challenges rarely encountered in the field of health care professionals. Although orthopedic surgeons are not considered as front-line fighters against COVID-19, they nevertheless have to play an important role in the face of an increasing psychological and emotional stress which comes from working in a post COVID era. The dread of getting infected while treating and operating on patients and the sinking feeling of spreading the contagion to their loved ones has put a massive toll on the emotional well-being of our orthopedic community and needs to be addressed through open and fair discussion. So, during these trying times, we need to rise to the occasion and work as a close knitted team to overcome every obstacle encountered in the face of COVID-19 pandemic.

Funding: No funding sources Conflict of interest: None declared

Ethical approval: The study was approved by the institutional ethics committee

\section{REFERENCES}

1. Ashford RU, Nichols JS, Mangwani J. Annotation: The COVID-19 pandemic and clinical orthopaedic and trauma surgery. $\mathrm{J}$ Clin Orthop Trauma. 2020;11(3):504-5.
2. Shi Y, Wang J, Yang Y, Wang Z, Wang G, Hashimoto $\mathrm{K}$ et al. Knowledge and attitudes of medical staff in Chinese psychiatric hospitals regarding COVID-19. Brain Behav Immun-Health. 2020;4:100064.

3. Chang Liang Z, Wang W, Murphy D, Po Hui JH. Novel Coronavirus and Orthopaedic Surgery: Early Experiences from Singapore. JBJS Latest Articles. 2020;102(9):745-9.

4. Viswanath A, Monga P. Working through the COVID-19 outbreak: Rapid review and recommendations for MSK and allied heath personnel. J Clin Orthop Trauma. 2020;11(3):500-3.

5. Awad ME, Rumley JCL, Vazquez JA, Devine JG. Peri-operative Considerations in Urgent Surgical Care of Suspected and Confirmed COVID-19 Orthopedic Patients: Operating rooms protocols and recommendations in the Current COVID-19 Pandemic. J Am Acad Orthop Surg. 2020;28(11):45163.

6. Tan Z, Phoon PHY, Zeng LA, Fu J, Lim XT, Tan TE et al. Response and Operating Room Preparation for the COVID-19 Outbreak: A Perspective from the National Heart Centre in Singapore. J Cardiothorac Vasc Anesth. 2020;34(9):2331-7.

7. Ducournau F, Arianni M, Awwad S, Baur E-M, Beaulieu J-Y, Bouloudhnine $M$ et al. COVID-19: Initial experience of an international group of hand surgeons. Hand Surg Rehabil. 2020;39(3):159-66.

8. Giacomo P, Damiano S, Elena D, Giulia B, Vincenzo S. CoViD-19 and Ortho and Trauma Surgery: the Italian Experience. Injury. 2020;51(6):1403-5.

9. Ahmed S, Leong Glenn TW, Chong Y-L. Surgical Response to COVID-19 Pandemic: A Singapore Perspective. J Am Coll Surg. 2020;230(6):1074-7.

10. Vaccaro AR, Getz CL, Cohen BE, Cole BJ, Donnally CJI. Practice Management During the COVID-19 Pandemic. J Am Acad Orthop Surg. 2020;28(11):46470.

11. Loeb AE, Rao SS, Ficke JR, Morris CD, Riley LHI, Levin AS. Departmental Experience and Lessons Learned with Accelerated Introduction of Telemedicine During the COVID-19 Crisis. J Am Acad Orthop Surg. 2020;55:164-6.

12. Parisien RL, Shin M, Constant M, Saltzman BM, Li $\mathrm{X}$, Levine $\mathrm{WN}$ et al. Telehealth Utilization in Response to the Novel Coronavirus (COVID-19) Pandemic in Orthopaedic Surgery. J Am Acad Orthop Surg. 2020;28(11):e487-92.

13. Lancaster EM, Sosa JA, Sammann A, Pierce L, Shen W, Conte $\mathrm{M}$ et al Rapid Response of an Academic Surgical Department to the COVID-19 Pandemic: Implications for Patients, Surgeons, and the Community. J Am Coll Surg. 2020;230(6):64-73.

14. Gan WH, Lim JW, Koh D. Preventing Intra-Hospital Infection and Transmission of Coronavirus Disease 2019 in Health-care Workers. Saf Health Work. 2020;11(2):241-3.

15. Ellis R, Hay-David AGC, Brennan PA. Operating during the COVID-19 pandemic: How to reduce 
medical error. $\mathrm{Br} \mathrm{J}$ Oral Maxillofac Surg. 2020;58(5):577-80.

16. Dunham AM, Rieder TN, Humbyrd CJ. A Bioethical Perspective for Navigating Moral Dilemmas Amidst the COVID-19 Pandemic. J Am Acad Orthop Surg. 2020;28(11):471-6.

17. Wang Y, Di Y, Ye J, Wei W. Study on the public psychological states and its related factors during the outbreak of coronavirus disease 2019 (COVID-19) in some regions of China. Psychol Health Med. 2020;8506:1-10.

18. Guo X, Wang J, Hu D, Wu L. The orthopedic forum survey of COVID-19 disease among orthopedic surgeons in Wuhan. People's Republic of China. 2020;1-15.

19. Lai J, Ma S, Wang Y. Factors associated with mental health outcomes among health care workers exposed to coronavirus disease 2019. JAMA Netw open. 2020;3(3)e203976.

Cite this article as: Muzzammil M, Jahanzeb S, Asghar A, Jabbar S, Waheed H. Impact of the COVID-19 pandemic on orthopedic surgeon in Pakistan. Int J Res Orthop 2021;7:12-8. 